\title{
BMJ Open Exploring how Brazilian immigrant mothers living in the USA obtain information about physical activity and screen time for their preschool-aged children: a qualitative study
}

\author{
Ana Cristina Lindsay, ${ }^{1,2}$ Carlos André Moura Arruda, ${ }^{3}$ \\ Márcia Maria Tavares Machado, ${ }^{3}$ Gabriela Pereira De Andrade, ${ }^{1}$ Mary L Greaney ${ }^{4}$
}

To cite: Lindsay AC, Moura Arruda CA, Tavares Machado MM, et al. Exploring how Brazilian immigrant mothers living in the USA obtain information about physical activity and screen time for their preschool-aged children: a qualitative study. BMJ Open 2018;8:e21844. doi:10.1136/ bmjopen-2018-021844

- Prepublication history for this paper is available online. To view these files, please visit the journal online (http://dx.doi. org/10.1136/bmjopen-2018021844).

Received 23 January 2018 Revised 18 July 2018 Accepted 19 July 2018

Check for updates

(C) Author(s) (or their employer(s)) 2018. Re-use permitted under CC BY-NC. No commercial re-use. See rights and permissions. Published by BMJ.

For numbered affiliations see end of article.

Correspondence to Dr Ana Cristina Lindsay; Ana.Lindsay@umb.edu

\section{ABSTRACT}

Objective To explore how Brazilian-born immigrant mothers living in the USA obtain information about physical activity (PA) and screen time (ST) behaviours for their preschool-aged children.

Research design Focus group discussions (FGDs) were used to gain an in-depth understanding of research topics. All FGDs were audio-recorded and professionally transcribed verbatim. The Portuguese transcripts were analysed using thematic analysis, an iterative process of coding the data in phases to create meaningful patterns.

Participants Thirty-seven Brazilian-born immigrant mothers of preschool-age children.

Setting This study was conducted in two cities in Massachusetts (MA). Participants were recruited from two predominantly Brazilian churches, local Brazilian businesses and community-based social and health services organisations in the Greater Boston area in MA. Results Analyses revealed that the mothers participating in this study did not initially actively seek out information about PA and ST for their preschool-age children, but that they received unsolicited information about these behaviours from multiple sources including their child's paediatrician, Women, Infant and Children (WIC) programme staff, members of their social network of Brazilian friends and the Brazilian media. Mothers reported that this unsolicited information increased their knowledge about the importance of making sure their children were physically active and not participating in excessive ST. This increased awareness led mothers to actively seek information about PA and ST behaviours via the internet and through interpersonal communication with fellow Brazilian friends and family.

Conclusions Given the value Brazilian immigrant mothers placed on the advice of their paediatricians and WIC staff, interventions should consider involving these healthcare professionals, possibly through including endorsement (eg, prescription for PA and maximum ST). More research is needed to ensure Brazilian immigrant mothers' health and media literacy including their ability to navigate the online environment and to discern the accuracy and quality of information from various web sites.

\section{Strengths and limitations of this study}

- This is the first qualitative study to our knowledge to explore how Brazilian-born immigrant mothers living in the USA obtain information about physical activity (PA) and screen time (ST) for their preschool-aged children.

- Study findings are based on a non-random and purposeful sample of low-income, Brazilian-born immigrant mothers in two Massachusetts (MA) communities which limits generalisability.

- There is a possibility of selection bias as mothers with a heightened interest in or awareness of the importance of child health behaviours may have been more likely to participate in the study.

- The use of snowball sampling to recruit participants might have resulted in the recruitment of study participants who share similar beliefs, attitudes and behaviours related to PA and ST.

- The present study included only mothers, and this is a limitation given increasing evidence suggesting the importance of including both parents in child health promotion and obesity prevention research and interventions.

\section{INTRODUCTION}

One in five children in the USA is a member of an immigrant family which is defined as a family with at least one immigrant parent. ${ }^{1} \mathrm{~A}$ large proportion of these children are part of immigrant families originally from Latin America $55 \%$ of all first-generation and second-generation immigrants in the USA were of Hispanic origin in 2014), making Latinos the largest and fastest growing immigrant group in the USA. ${ }^{1}$

Brazilians are a rapidly increasing Latino immigrant US population subgroup, yet little research has focused on health-related behaviours that may affect Brazilian immigrant children's health. ${ }^{23}$ Although Brazilians 
share many cultural characteristics of other Latin American population groups, they represent many different ethnic backgrounds, including Africans, Europeans and native Brazilians. ${ }^{2}{ }^{3}$ Portuguese is the official language of Brazil, and it is an important cultural difference between Brazilians and other Latin American population groups that primarily speak Spanish. ${ }^{4}$ According to the 20062010 American Community Survey of the approximately 400000 Brazilian immigrants living in the USA, nearly half live in the Northeastern states, mostly in Massachusetts (MA; about 19\%), New York and New Jersey. ${ }^{2}{ }^{3}$ A recent study conducted in the Greater Boston area of MA found that $48.2 \%$ of Brazilian immigrant children (aged 3-12 years) were overweight or obese. ${ }^{5}$

Promoting physical activity (PA) is a key component of preventing and reducing childhood obesity. ${ }^{6-8}$ Unfortunately, rates of PA among young children have declined over the past decades, and most children are not accruing sufficient daily PA. ${ }^{6-8}$ Excessive sedentary behaviour (eg, screen time) is a greater problem among racial/ethnic minority children in the USA, with greater prevalence of sedentary behaviours among Hispanic children than non-Hispanic white children. ${ }^{7}$

In the USA, the National Association for Sport and Physical Education recommends that preschoolers engage in at least $60 \mathrm{~min}$ of structured PA and $60 \mathrm{~min}$ of unstructured, or spontaneous active play daily for a combined minimum of $120 \mathrm{~min}$ of PA daily. ${ }^{8}$ Similarly, the American Academy of Pediatrics (AAP) recommends that clinicians encourage parents to increase their children's PA and decrease the time their children spend in sedentary activities (eg, screen time, time spent indoors, etc) in a manner compatible with the developmental level of a child. ${ }^{9}$ In addition, due to the increasing rates of ST among preschool-aged children, ${ }^{9-11}$ several countries including the USA have developed ST guidelines. For example, the recently updated AAP guidelines recommend that ST should not exceed 1 hour per day for children aged 2-5 years. ${ }^{9}$

Mounting evidence points to the unique and central role parents play in helping their children develop early healthy PA and ST behaviours. ${ }^{12-25}$ One way parents influence their children's PA and ST behaviours is through their parenting practices. ${ }^{14-16} 18$ Parents' access to information about PA and ST behaviours that is accurate and that they easily understand is essential to their ability to promote healthy PA and ST behaviours for their children. ${ }^{25-27}$ Access to comprehensible information is especially important for minority low-income parents who may face additional barriers (eg, limited or lack of access to quality healthcare, language barriers, etc) to obtaining health-related information that they understand. ${ }^{28} 29$

Despite the importance of parents' access to accurate information about PA and ST behaviours for their young children, there is little available research examining how and from whom low-income, immigrant parents seek and obtain this information. ${ }^{29}$ While current research indicates that parents receive health-related information from a range of sources including books, magazines, the internet, family, friends and health professionals, ${ }^{30-32}$ no existing research, to our knowledge, has examined how Brazilian-born immigrant mothers' living in the USA access information about PA and ST behaviours for their young children. This information is crucial for the design of culturally appropriate early childhood obesity prevention interventions tailored to this ethnic group. Therefore, the aim of this exploratory study was to investigate how Brazilian-born immigrant mothers living in the USA obtain information about PA and ST behaviours for their preschool-aged children.

\section{METHODS}

\section{Design, setting and sample}

This study was conducted in two cities in MA: Somerville and Everett. The present qualitative study was part of a larger ongoing mixed-methods research study (to date 113 unique families) with Brazilian families living in the Greater Boston, MA, examining parenting styles and parenting practices (eg, promoting healthy eating, PA and sleep, and limiting ST) related to the risk of childhood obesity. ${ }^{33-35}$

Focus group discussions (FGDs) were used to gain an in-depth understanding of how Brazilian-born immigrant mothers living in the USA obtain information about PA and ST behaviours for their preschool-aged children. FGDs are valuable techniques for working in diverse cultural settings as they yield rich information ${ }^{36}$ as the synergistic effects of the group settings elicit ideas and discussion that may not arise in individual interviews. ${ }^{37}$

\section{Patient and public involvement}

This study did not involve any patients. Public involvement in this study was limited to: (1) collaboration of community members on posting of flyers at local Brazilian businesses and community health services organisation and public announcements at masses and events at local and predominantly Brazilian churches in the Greater Boston area and (2) participation in FGDs.

\section{Data collection}

A convenience sample of mothers was recruited between March and August 2017 from two predominantly Brazilian churches, local Brazilian businesses and community-based social and health services organisations in the Greater Boston area, MA. Participants were recruited through flyers posted at local Brazilian businesses and community-based social and health services agencies, as well as through announcements and events at predominantly Brazilian churches. Interested participants called the phone number listed on the flyer or spoke to study staff at church events. Study staff assessed eligibility, and women were eligible to participate if they had at least one child aged 2-5 years, were of Brazilian ethnicity, born in Brazil and had been living in the USA for at least 12 months. In addition, participants were recruited using a 
snowball technique, ${ }^{38}$ with women enrolled in the study asking their Brazilian friends with preschool-aged children if they would be interested in participating in the study. ${ }^{38}$

Seven FGDs (range of 4-7 participants per FGD) with a total of 37 Brazilian immigrant mothers were held at the two local churches between April and August 2017. Of the 37 mothers, seven (approximately 19\%) were recruited through the use of snowball sampling technique.

Before each FGD, the moderator explained in Portuguese the study's purpose, FGD procedures and study confidentiality. A native Brazilian-Portuguese speaker (ACL) trained in qualitative research methods moderated all FGDs in Portuguese using a semistructured discussion guide that explored participants': (1) information-seeking about PA and ST and (2) sources of information about PA and ST behaviours for their young children. The guide also explored mothers' beliefs, attitudes and practices related to PA, ST, sleep and bedtime routines. Results from these topics are presented elsewhere. ${ }^{39}$

The guide was piloted in an FGD with a small group of Brazilian immigrant mothers $(n=4)$ and then refined (eg, rewording some questions and changing the order of some questions) prior to use. Data from the pilot FGD were not included in the present study.

Before each FGD started, participants were asked to think about their preschool-aged children when participating in the discussion. A trained, bilingual (Portuguese and English) research assistant (GDA) took notes during all FGDs, which were audio-recorded and lasted between 60 and $80 \mathrm{~min}$. The moderator and research assistant met for about 15 min after each FGD to identify new and review recurring themes which were entered into a grid that was used to closely follow emerging themes and to determine when data saturation was reached.

Lastly, at the end of each FGD, participants completed a brief, self-administered questionnaire in Portuguese that assessed education, marital status, access to healthcare services including participation in government-sponsored health and nutrition programmes (eg, WIC, Supplemental Nutrition Assistance Program, etc), country of origin, length of time living in the USA and acculturation which was assessed via the Short Acculturation Scale for Hispanics (SASH), a 12-item measure scale validated for use in Latinos, including Mexican-Americans, Cuban-Americans, Puerto Ricans, Dominicans and Central and South Americans. The SASH assesses language use, media use and ethnic social relations, ${ }^{40}$ and items are measured on a scale of 1-5 (1=least acculturated, $5=$ fully acculturated), and an acculturation score was computed by averaging across the 12 items.

\section{Analysis}

A professional transcriptionist and native Brazilian speaker transcribed all audio-recordings verbatim. The Portuguese transcripts were analysed using thematic analysis, an iterative process of coding the data in phases to create meaningful patterns ${ }^{41-43}$ by two experienced qualitative researchers who are native Portuguese speakers (ACL, CAMA). Each researcher read several transcripts numerous times to become familiar with the content and generate initial codes. ${ }^{42} 43$ The researchers then manually coded transcripts independently, but met regularly to discuss coding and to identify and resolve disagreements in coding. ${ }^{43}$ The coded text describing similar ideas were grouped and sorted to identify emergent themes and subthemes. Finally, salient text passages were extracted and translated into English to be used as illustrative quotes for the emergent themes. Descriptive statistics and frequencies were calculated for data collected in the sociodemographic survey using Microsoft Excel 2008.

\section{RESULTS}

Seven FGDs were conducted before saturation was reached, with no new themes or subthemes emerging during the final group. Mothers' $(n=37)$ ages ranged from 26 to 41 $($ mean $=35.3, \mathrm{SD}=2.8)$. The majority of participants were married (92\%; n=34), and approximately $89 \%$ had two children $(n=33)$. Most $(72 \% ; n=21)$ had graduated from high school and owned their own housecleaning business (92\%; n=34). Approximately half $(51 \% ; n=19)$ reported a family income of $\$ 40000$ or less, which in the USA is considered low-income for a family of four, while the remainder reported an annual income between $\$ 40000$ and \$60 000 which is considered to be a low-middle income. All mothers reported having access to healthcare services through government-sponsored insurance (MassHealth $89.2 \%, \mathrm{n}=33$ ) or private health insurance through work $(10.8 \%, n=4)$. In addition, mothers reported that they regularly took their children for healthcare visits (eg, well and sick visits) and public health and nutrition services (eg, WIC).

Participants were originally from three main regions of Brazil (eg, the Southeast (eg, Espirito Santo, Sao Paulo and Minas Gerais), the South (eg, Santa Catarina), the Midwest (eg, Goias and Mato Grosso)), with the majority $(64.7 \%$; $n=22)$ being from the state of Minas Gerais, in the Southeast region. In addition, the majority spoke Portuguese at home $(92 \%, n=34)$, watched television (TV) programmes in Portuguese $(95 \%)$ and reported that the majority of their friends were Brazilians (87\%). Mothers had lived in the USA for an average of $6.7(\mathrm{SD}=2.84)$ years, and their mean acculturation score was $1.43(\mathrm{SD}=0.77)$, indicating that they identified more closely with Brazilian culture than with that of the USA.

Emergent themes identified in the analyses are presented below with representative quotes.

Theme 1: mothers report that they did not initially actively seek information about PA and ST for their preschool-aged children

Most mothers reported that initially they did not actively seek information about PA and ST for their preschool-age 
children. Some mothers said they had not thought about finding information about PA. Other mothers, however, explained that they did not seek out this information as they felt it was not needed because either they were knowledgeable about PA or because their children were active. In fact, several mothers mentioned that young children are 'naturally' physically active and therefore, they did not think it was necessary to seek advice and/or information about PA for their young children.

...In my case, I have an older one [9 years old], so I never think of asking or looking for information about PA for the little one... Mother \#16, two children (9 and 4years old)

... I don't think that there is a need to worry about young children being physically active. They [young children] are always moving and busy ... Mother \#3, two children ( 8 and 3 years old)

Although several mothers felt that their preschool-age children had too much ST (eg, TV watching and use of electronics), they did not think to seek out information about ST. Mothers explained that they did not seek out information because although they recognised a lot of ST could be problematic, most felt ST was an acceptable part of children's daily lives, even young children.

... In reality, we all know that it's not good for the kids to be on the iPad all the time... Mother \#9, three children (10, 7 and 3years old)

...Nowadays it is normal for little kids, even babies to be 'on screen' and have electronic toys to watch cartoons... even babies, as soon as they can hold, they have an iPhone or an iPad on their hands... that's how kids are growing these days... and it's not only in the United States, it's everywhere. In Brazil it's the same... it's a problem, but it's how it is nowadays. Mother \#22, two children (6 and 4 years old)

\section{Theme 2: mothers receive unsolicited information about PA and ST from multiple sources}

Analyses revealed that mothers receive unsolicited information about PA and ST behaviours from three main sources: (1) healthcare professionals (paediatricians and WIC staff), (2) interpersonal communication with their social network of Brazilian friends and family members and (3) Brazilian media. In addition, public health education campaigns and community-based programmes and health-related research were identified as sources of information. As discussed below, many mothers spoke of these information sources prompting them to seek additional information about PA and ST.

\section{Healthcare professionals (paediatricians and WIC staff)}

More than half of the mothers reported that their child's paediatrician was an important source of unsolicited information about PA and ST behaviours. Mothers said that they did not initially seek out information about these behaviours from their paediatrician, but almost all reported that their paediatrician inquired about their child's PA and ST behaviours, and shared information on current recommendations during well-child appointments.

When my son was about 2-3 years old, I recall his pediatrician asking some questions about his screentime habits... like did he have a TV in his [son] bedroom? How much TV he watched? Mother \#28, one child (4years old)

I remember one time I took my daughter to a doctor's appointment and she had several scratches and bruises in her legs and the doctor was looking... I felt nervous and started explaining that she's always running around non-stop and she [doctor] said that's good sign that she's active and then she said it was important for children to be physically active and at the end of the visit gave me some printed information... Mother \#36, three children (7, 5 and 3 years old)

For many mothers, the information from their children's doctor served as a catalyst for them to seek out additional information from their social networks of friends and family.

Until her [daughter's] doctor mentioned about too much TV and use of electronics being a problem and interfering with sleep I did not think to look for information, but after he [doctor] mentions it I started talking to my friends who also have children and that's how I started learning... Mother \#5, one child (5years old)

... in a doctor's visit my son's pediatrician asked me about how much TV and other electronics he typically watched ... that got me thinking that perhaps my son was watching too much TV and videos. So, I started thinking about it more and talking with others [friends] and looking for information ... he was indeed spending a lot of time watching videos and playing games... Mother \#19, two children (7 and 4 years old)

Nearly all mothers reported valuing the information they received from their paediatricians. Moreover, some mothers spoke of using repeated well-child visits as opportunities to clarify or validate PA and ST information obtained from other sources (eg, friend and the internet).

In my case, I like to ask her doctor because you hear and read about all sorts of health information everywhere. Sometimes it's hard to know what's [information] good and what's not, right? So, when I take her to her medical consultation I ask her doctor because he is the expert...I feel confident on what he says. He's really good. Mother \#7, one child (4years old)

In addition, some mothers also mentioned that programme staff at the WIC was a valued source of information about PA and ST recommendations for their 
preschool-age children. As with their child's paediatrician, mothers mentioned that they did not seek out this information from WIC staff rather this information, especially information about ST (eg, TV viewing) was given during routine visits.

Every time I take my daughter for her WIC visit, the nurse [WIC staff] asks about her foods and what she eats... she also always ask questions about the TV. It's not really about physical activity, it's more how much TV she watches ... she talks a lot that kids should not watch a lot of TV and have TV in the bedroom. Every time I go there [WIC program], she asks and talks about that... Mother \#9, two children (7 and 4 years old)

Every time I take my daughter to her WIC appointment the WIC nurse [WIC staff] asks about her eating, TV watching and if she spends too many hours watching TV or videos ... Mother \#33, three children (11, 8 and 3years old)

\section{Interpersonal communication with social network members}

Several mothers mentioned that their conversations with fellow Brazilian immigrant mothers and their family members made them to take notice of their young child's PA and ST behaviours and to seek out PA-related information, including information about existing PA programmes, classes and so on.

[child's name] is my first child and I did not think much about his physical activity until a friend at church mentioned that she had enrolled her 3 year old son in a movement class at the YMCA. That got me curious and thinking about my son and led me to seek further information... Mother \#17, one child (3years old)

Mothers spoke of valuing information from their friends and family due to their similar cultural backgrounds, experiences, and shared beliefs and values. In addition, mothers valued the ease of communication due to speaking in their native language, Portuguese. Moreover, several mothers mentioned that their social networks provided a broad range of information about PA and ST, including information about managing ST and organised sports and classes for young children.

We [mothers] always talk among ourselves about our children's health and health habits... we [mothers] all struggle with the kids using the iPads too much and playing games, watching videos, so we kind of inform each other of what we know... Mother \#22, two children (6 and 4 years old)

My son is 5 years old and all he wants to do is to watch videos and play games on his iPad... So, I start talking with my friends who also have children his age, asking to see if they had some suggestions about managing their children's use of electronics because I have to say that in my house it's a struggle to get him [son] to want to do something else. Mother \#31, two children (6 and 4 years old)

.... a friend of mine told me that her son [4years old] was doing an all ball sports class (soccer, basket ball) at the local YMCA. So I signed my son up. He loves it! He's always asking me, 'mom, do I have sports class today? Mother \#12, one child (4years old)

Mothers felt that being able to discuss information about PA and ST in their native language with other Brazilian mothers was particularly beneficial. Several mothers reported valuing the opinion of their friends who were mothers like them, and felt a sense of social support from their interactions with friends.

I don't know, it's just easier to talk in one's own language. We understand each other and know exactly what everything means... even if you know some English, it's not the same... Mother \#34, three children (9, 7 and 3 years old)

It's helpful to hear from other mothers like us, who are also trying to manage a work schedule, raising a family, and trying to make sure that the children are healthy...I am very thankful that I have friends...like, from Brazil, who understand the way we raise children. You know, it's nice when you are from the same culture... It's good to know that you are not alone... Mother \#27, two children ( 7 and 5 years old)

Additionally, mothers reported that the information and advice from friends and family influenced their parenting practices including enrolling children in sports and limiting ST.

A friend of mine who has three children, one the same age as my son, mentioned that she had her two sons ( 6 and 3 years old) enrolled in karate lessons and that her sons loved it! She [friend] said it was really good for her sons, even the little one. So, I decided to enrol my son... and he loves it! Mother \#6, one child (4 years old)

...in my house now I have a rule - no iPad at the table during meals! I was talking with my friend and she said that she set rules for her children because otherwise the kids are on their iPad all day long. I think that's a great idea, and I now do the same. Mother \# 13 , two children (8 and 4 years old)

\section{Brazilian media}

About half of the mothers mentioned learning about PA and ST behaviours from media outlets including Brazilian TV channels that aired Brazilian programmes. Several mothers viewed these media outlets as providing information that was easy to grasp due to its cultural and linguistic relevance.

I watched a program on Globo [Brazilian TV channel] about childhood obesity and it had lots of good information about children's unhealthy habits... we live here in America and sometimes we think that 
in Brazil things are the same way when we [parents] were kids, but things have changed... it's the same as here in America...kids not being active, a lot of use of electronics ... Mother \#11, two children ( 6 and 4 years old)

Sometimes on Bem Estar ['Well Being'- Brazilian TV show] they have interviews and talk about children's health. So, I hear the information and then I talk with friends, other moms, you know, asking if they heard, if they know... we [mothers] talk about all sorts of things regarding our children amongst ourselves... and we are always learning from each other... Mother \#34, two children (9 and 5 years old)

\section{Public health campaigns}

A few mothers mentioned obtaining information about PA and ST from public health campaigns on TV, radio, billboards and so on, and that these campaigns were informative, and increased their awareness and knowledge about the importance of these behaviours for young children which in turn led to their seeking additional information on these topics.

You hear about children's health everywhere... they have a lot of information on programs... you even see it in billboards on the road. Here [USA] there is a big concern about childhood obesity. Everywhere you turn you find information about it... Mother \#35, three children (12, 9 and 4 years old)

\section{Community-based programmes and health-related research}

A couple of mothers mentioned that community-based programmes and health-related research offered by faith-based organisations and at other Brazilian community events as being sporadic sources of information for families.

...in community events sometimes we [mothers] hear about childhood obesity-nutrition, physical activity, and too much use of electronics by kids... a lot of the community health fairs have information on children's health, and a lot about child obesity ... Mother \#22, two children (6 and 4years old)

A couple of years ago they had a research study at our Church... I think it was called Viver Bem (Live Well)? They [research staff] came several times to talk to the mothers' groups. They talked about obesity, and families with children. They ask a lot questions too and then if you wanted you could participate $[$ research]... it was a lot related to preventing obesity in children and they talked about physical activity and electronics and TV too... Mother \#4, four children $(9,7,5$ and 3 years old)

\section{Theme 3: mothers use the internet to seek out information about PA and ST in their native language}

The internet emerged as the main information source used by mothers to seek out information about PA and ST.
Almost three-quarters of the mothers reported accessing the internet for the range of available information in their native language. Women accessed the internet via multiple platforms (computers, smartphones, tablets), with smartphones being used most often due to ease and convenience.

Whenever I have questions, I ask Dr. Google (laughs)! The best thing is Dr. Google speaks my language [Portuguese] (more laughs). My husband sometimes asks me how do I know something is right or not? I say, I don't really know, so let's ask Dr. Google! (laughs). Mother \# 13, two children (8 and 4 years old)

Nonetheless, several mothers also mentioned feeling overwhelmed with abundance of information available via the internet, and that they were not always able to discern between 'good' and 'bad' information.

You start looking for information and there's just so much out there that it's easy to get lost...it's hard to know what's good information and what's not...I like that one can find out about anything in the Internet, but when it's health I think one needs to be more careful... Mother \# 28, two children (7 and 3 years old)

As mentioned previously (see the Healthcare professionals (paediatricians and WIC staff) section), several mothers reported validating information that they obtained from the internet with their children's paediatrician.

Whenever the doctor mentions something about screen time or physical activity during a health visit I take the opportunity to clarify any questions I might have. You know, there's just so much information out there. I like to hear what is the doctor's [paediatrician] opinion, so I ask... Mother \#7, one child (4 years old)

\section{DISCUSSION}

Parents play a unique and central role in promoting their children's early development of healthy PA and ST behaviours, ${ }^{13-24}$ and it is important to consider how parents access information related to these behaviours. Therefore, the current study explored how Brazilian immigrant mothers living in the USA obtain information about PA and ST behaviours for their preschool-age children. This information is needed due to the dearth of research on how low-income immigrant mothers obtain information about PA and ST behaviours for their young children.

Mothers in this study reported that they did not initially seek out information about PA and ST for their preschool-age children, but that they received unsolicited information from multiple sources (eg, healthcare professionals, social network of Brazilian friends and family, Brazilian media, etc) that prompted them to seek 
out additional information about these behaviours. Most of the Brazilian immigrant mothers participating in this study did not perceive their preschool-aged children's PA level as being problematic because they were active and healthy. However, almost all mothers spoke of excessive ST as being a concern for their preschool-aged children, but also voiced an acceptance of ST being part of their preschool-aged children's lives.

Routine encounters with paediatricians and WIC staff were identified as important sources of unsolicited information about PA and ST behaviours for mothers in the current study. Similar to previous research, ${ }^{44-46}$ study findings suggest that routine well-child visits increased mothers' awareness of the importance of early PA and ST behaviours for their children's health and well-being, and this realisation motivated mothers to seek out additional information from other sources. ${ }^{44-46}$ Study findings also showed that mothers valued information provided by their child's paediatrician and WIC staff, and that many took advantage of routine health encounters with their child's paediatrician to corroborate information obtained from other sources. This finding concurs with evidence documenting that healthcare professionals play a central role in guiding consumers to quality online health information. ${ }^{47}$ Given the value mothers placed on the unsolicited and solicited advice they receive from their paediatricians and WIC staff, interventions should consider involving these healthcare professionals possibly through including endorsement (eg, prescription for PA and maximum ST). In addition, paediatricians and WIC staff could ask mothers during routine visits about their use of the internet to obtain information and provide some anticipatory guidance on accurate information related to PA and ST behaviours of preschool-aged children.

Consistent with previous research with other ethnic minority groups including Latinos, ${ }^{29} 49$ we found that interpersonal communication between social network members composed of ethnically similar friends and family members was a key source for mothers' obtaining information about PA and ST for their preschool-age children. ${ }^{29}$ Prior research shows that shared language, cultural beliefs and values are important factors influencing reliance on interpersonal communication with ethnically similar social networks of friends and family. ${ }^{50}$ Mothers reported that speaking with other Brazilian mothers in their native language was particularly important and that they valued the information and advice about PA and ST they received. This finding is important and suggests that interventions should consider messages that address the influence from ethnic-like social support networks of Brazilian immigrant mothers. ${ }^{50}{ }^{51}$ Interventions also could be designed to include social network members. For example, participants could be asked to enrol in a programme with their best friend or mother.

Results of this study revealed that the internet is an important source of information for mothers seeking information about PA and ST behaviours for their preschool-aged children. ${ }^{52-55}$ This finding is in agreement with previous research with other ethnic immigrant populations. ${ }^{46} 56$ Nonetheless, study findings also indicate that several mothers felt overwhelmed with the abundance of available information and were not always able to discern the credibility of this information. In combination, these findings suggest the potential for further exploration of e-health interventions (email, text, etc) that also include a media literacy component to enable mothers to evaluate information sources to disseminate PA and ST information to Brazilian immigrant families with young children. Furthermore, although more research is needed, study findings suggest that increasing the availability of reliable and valid e-health information in Portuguese language could have a positive influence on increasing awareness and promoting healthy PA and ST behaviours among low-income, minority Brazilian children of immigrant families living in the USA. Future research should assess Brazilian immigrant mothers' health-literacy levels and the mothers' ability to navigate the online environment including discerning trustworthy information sources and evaluating the quality of web sites. $^{5859}$

In this study, TV programmes in the mothers' native language (Portuguese) emerged as an important source of information about PA and ST behaviours. Several mothers reported appreciating that these programmes offered culturally relevant information that aligned with their beliefs and values. This finding is supported by prior research with other ethnic minority. ${ }^{60}{ }^{61}$ Interventions designed for immigrant Brazilian mothers living in the USA should take into account this potential source of information, and as much as possible assess the content of the most popular health-related programmes watched by Brazilian families to deliver information that is relevant and culturally attuned with Brazilian culture and values, mothers' health and media-literacy levels, and that provides sound, state-of-the-art scientific information to this ethnic group.

Some mothers reported that public health campaigns provided information about PA and ST behaviours. Nevertheless, similar to previous studies, ${ }^{60-62}$ for some mothers, language barriers ${ }^{62}$ appeared to influence their preference for obtaining information from other sources such as the internet and Brazilian TV shows. Moreover, with the exception of advice received from healthcare professionals, mothers in this study reported a preference for accessing information about PA and ST in their native language, Portuguese. This finding is important and suggests that successful interventions will require attention to mothers' language preference for delivery of information. These findings combined suggest that increased availability of campaigns in Portuguese and partnering with Brazilian ethnic media could be viable strategies to disseminate health information to Brazilian immigrant families living in the USA.

A couple of mothers also spoke of community-based programmes and health-related research as sources of 
information about PA and ST behaviours. This finding is noteworthy and emphasises the significance that community-based programmes and health-related research can play in raising awareness and educating minority community groups about relevant health-related topics, despite only a couple of mothers mentioned these sources.

The mothers participating in this study had low acculturation levels and identified more closely with Brazilian culture than with US culture. Previous research with other Latino population groups suggests that acculturation level is an important influence on health information-seeking behaviour and preferred sources of health information. ${ }^{63}$ More-acculturated individuals are more likely to seek health information and more likely to use electronic channels than less-acculturated individuals who are more likely to rely on social networks of friends for obtaining health information. ${ }^{6364}$ Our finding regarding Brazilian immigrant mothers' low acculturation levels, combined with results of previous research suggests that successful interventions targeting Brazilian immigrant parents will require consideration of the target population's acculturation levels.

Finally, study findings suggest the importance of involving healthcare professionals (paediatricians and WIC staff) in interventions. Healthcare professionals could endorse healthy behaviours (eg, prescription of PA and limiting ST) as well as information delivered by e-health interventions which based on our findings would likely increase both legitimacy and acceptance of such interventions aimed at increasing awareness and educating Brazilian immigrant parents about PA and ST behaviours for their preschool-aged children. ${ }^{65}$

\section{LIMITATIONS AND STRENGTHS}

Study results should be considered in light of study limitations. Findings are based on a non-random and purposeful sample of low-income, Brazilian-born immigrant mothers in two MA communities which limits generalisability. There is a possibility of selection bias as mothers with a heightened interest in or awareness of the importance of child health behaviours may have been more likely to participate in the study. Moreover, the use of snowball sampling to recruit participants might have resulted in the recruitment of study participants who share similar beliefs, attitudes and behaviours related to PA and ST. Thus, further research is needed to increase generalisability and to explore whether results apply to a broader group of Brazilian immigrants. In addition, this study did not objectively assess mothers' PA and ST behaviours. This is a limitation given that evidence suggests that parents' PA and ST behaviours influence their children's behaviours. Finally, the present study included only mothers, and this is a limitation given the increasing evidence suggesting the importance of including both parents in child health promotion and obesity prevention research and interventions. ${ }^{6-68}$ Future research can address these limitations by exploring information-seeking behaviours and sources of information used by low-income, minority mothers and fathers from other communities across the USA, selecting a larger sample size and employing multiple data-collection methods, including both qualitative and quantitative methods, and objectively assessing parents' and children's PA and ST behaviours.

\section{CONCLUSIONS}

Evidence suggests that parents need to be aware of the importance of early healthy behaviours and have access to accurate information that they easily understand so that they can implement healthy practices and routines for their young children. Study results indicate that despite mothers' reporting of widespread use of the internet to obtain information in their native language, routine healthcare visits with paediatricians emerged as an important source of information about PA and ST and mothers valued advice given by their child's paediatricians. Furthermore, mothers viewed WIC programme staff as providing important information about PA and ST. Given the value Brazilian immigrant mothers placed on the advice of their paediatricians and WIC professionals, interventions should consider involving these healthcare professionals possibly through including endorsement (eg, prescription for PA and maximum ST). Furthermore, given this study finding of mothers' reporting of widespread use of the internet to obtain information in their native language, culturally and linguistically suitable e-health interventions may be viable ways to provide accurate and easily understood information about PA and ST to Brazilian immigrant families with preschool-aged children living in the USA.

Finally, more research is needed to ensure Brazilian immigrant mothers' health and media literacy including their ability to navigate the online environment and to discern the accuracy and quality of information from various websites.

\section{Author affiliations}

${ }^{1}$ Department of Exercise and Health Sciences, University of Massachusetts, Boston, Massachusetts, USA

${ }^{2}$ Department of Nutrition, Harvard T.H. Chan School of Public Health, Boston, Massachusetts, USA

${ }^{3}$ Department of Community Health, School of Medicine, Federal University of Ceará, Fortaleza, Brazil

${ }^{4}$ Health Studies and Department of Kinesiology, University of Rhode Island, Kingston, Rhode Island, USA

Acknowledgements We thank the women who participated in the study and the personnel at the two churches who assisted with study recruitment.

Contributors ACL participated in study design, data collection, data analysis and manuscript preparation and review. CAMA participated in qualitative data analysis, manuscript preparation and manuscript review. MMTM participated in manuscript preparation and review. GPDA participated in data collection and manuscript preparation and manuscript review. MLG participated in study design, manuscript preparation and manuscript review.

Funding This study was supported by a grant from the Aetna Foundation Inc. (Grant no. 11-02395), for which Ana Cristina Lindsay, DDS, MPH, Dr.PH is principal investigator.

Competing interests None declared. 
Patient consent Not required.

Ethics approval This study was approved by the Institutional Review Board for the Protection of Human Subjects at the University of Massachusetts Boston.

Provenance and peer review Not commissioned; externally peer reviewed.

Data sharing statement Data and all other materials for this study are kept at the Department of Exercise and Health Sciences, University of Massachusetts Boston. The datasets generated during and/or analysed during the current study are not publicly available due the terms of consent to which participants agreed to, but are available from the corresponding author on reasonable request.

Open access This is an open access article distributed in accordance with the Creative Commons Attribution Non Commercial (CC BY-NC 4.0) license, which permits others to distribute, remix, adapt, build upon this work non-commercially, and license their derivative works on different terms, provided the original work is properly cited, appropriate credit is given, any changes made indicated, and the use is non-commercial. See: http://creativecommons.org/licenses/by-nc/4.0/.

\section{REFERENCES}

1. Hernandez DJ, Denton NA, McCartney SE. Children in immigrant families: looking to America's future. Social Policy Report 2008;22.

2. Lima A, Siqueira CE. Brazilians in the U.S. and Massachusetts: a demographic and economic profile: Gastón Institute Publications, Paper 50, 2007.

3. Jouët-Pastré C, Braga LJ. Becoming Brazuca: Brazilian Immigration to the United States. Cambridge, Mass: David Rockefeller Center for Latin American Studies, Harvard University Press, 2008.

4. Goza F. Brazilian immigration to North America. Int Migr Rev 1994;28:136-52.

5. Tovar A, Hennessy E, Must A, et al. Feeding styles and evening family meals among recent immigrants. Int J Behav Nutr Phys Act 2013;10:84

6. O'Dwyer MV, Fairclough SJ, Knowles Z, et al. Effect of a family focused active play intervention on sedentary time and physical activity in preschool children. Int J Behav Nutr Phys Act 2012;9:1-13.

7. Chuang RJ, Sharma S, Skala K, et al. Ethnic differences in the home environment and physical activity behaviors among low-income, minority preschoolers in Texas. Am J Health Promot 2013;27:270-8.

8. National Association for Sport and Physical Education (NASPE). Active start: a statement of physical activity guidelines for children from birth to age 5. 2nd edn. Sewickley, PA: American Alliance for Health, Physical Education, Recreation, and Dance, 2009.

9. Council On Communications And Media. Media and young minds. Pediatrics 2016;138:e20162591.

10. Commonwealth of Australia, Department of Health and Ageing. Move and play every day: National physical activity recommendations for children 0 to 5 years. 2010.

11. Tremblay MS, Leblanc AG, Carson V, et al. Canadian physical activity guidelines for the early years (aged 0 to 4 years). Applied Physiology, Nutrition, and Metabolism 2012;37:370-91.

12. Lindsay AC, Sussner KM, Kim J, et al. The role of parents in preventing childhood obesity. Future Child 2006;16:169-86.

13. O'Connor TM, Cerin E, Lee RE, et al. Environmental and cultural correlates of physical activity parenting practices among Latino parents with preschool-aged children: Niños Activos. BMC Public Health 2014;14:707.

14. Byrd-Bredbenner C, Martin-Biggers J, Povis GA, et al. Promoting healthy home environments and lifestyles in families with preschoo children: HomeStyles, a randomized controlled trial. Contemp Clin Trials 2018:64:139-51.

15. O'Connor TM, Cerin E, Hughes SO, et al. What Hispanic parents do to encourage and discourage 3-5 year old children to be active: a qualitative study using nominal group technique. Int J Behav Nutr Phys Act 2013;10:93.

16. O'Connor TM, Chen TA, Baranowski J, et al. Physical activity and screen-media-related parenting practices have different associations with children's objectively measured physical activity. Child Obes 2013:9:446-53.

17. Cespedes EM, McDonald J, Haines J, et al. Obesity-related behaviors of US- and non-US-born parents and children in lowincome households. J Dev Behav Pediatr 2013;34:541-8.

18. Downing KL, Hinkley T, Hesketh KD. Associations of parental rules and socioeconomic position with preschool children's sedentary behaviour and screen time. J Phys Act Health 2015;12:515-21.

19. Pearson N, Salmon J, Crawford D, et al. Are parental concerns for child TV viewing associated with child TV viewing and the home sedentary environment? Int J Behav Nutr Phys Act 2011;8:102.
20. Jago R, Sebire SJ, Edwards MJ, et al. Parental TV viewing, parental self-efficacy, media equipment and TV viewing among preschool children. Eur J Pediatr 2013;172:1543-5.

21. Jago R, Stamatakis E, Gama A, et al. Parent and child screenviewing time and home media environment. Am J Prev Med 2012;43:150-8.

22. Zecevic CA, Tremblay L, Lovsin T, et al. Parental influence on young children's physical activity. Int J Pediatr 2010:468-526.

23. Lindsay AC, Greaney ML, Wallington SF, et al. A review of early influences on physical activity and sedentary behaviors of preschoolage children in high-income countries. J Spec Pediatr Nurs 2017;22:e12182.

24. Smith BJ, Grunseit A, Hardy LL, et al. Parental influences on child physical activity and screen viewing time: a population based study. BMC Public Health 2010;10:593.

25. Finkelstein DM, Petersen DM, Schottenfeld LS. Promoting children's physical activity in low-income communities in colorado: what are the barriers and opportunities? Prev Chronic Dis 2017;14:e134.

26. Hammersley ML, Jones RA, Okely AD. Time2bHealthy - an online childhood obesity prevention program for preschool-aged children: a randomised controlled trial protocol. Contemp Clin Trials 2017;61:73-80.

27. Marsh S, Foley LS, Wilks DC, et al. Family-based interventions for reducing sedentary time in youth: a systematic review of randomized controlled trials. Obes Rev 2014;15:117-33.

28. Stanley RM, Jones RA, Cliff DP, et al. Increasing physical activity among young children from disadvantaged communities: study protocol of a group randomised controlled effectiveness trial. BMC Public Health 2016;16:1095.

29. Davis RE, Cole SM, McKenney-Shubert SJ, et al. An exploration of how mexican american wic mothers obtain information about behaviors associated with childhood obesity risk. J Nutr Educ Behav 2017:49:187-95.

30. Rooks RN, Wiltshire JC, Elder K, et al. Health information seeking and use outside of the medical encounter: Is it associated with race and ethnicity? Soc Sci Med 2012;74:176-84.

31. Brown A, Lopez MH, libraries P. Public libraries and Hispanics: immigrant hispanics use libraries less, but those who do appreciate them the most pew research center. Washington, DC, 2015:1-38.

32. Powe BD. Health information seeking among Rural African Americans, Caucasians, and Hispanics. Nurs Clin North Am 2015;50:531-43.

33. Lindsay AC, Wallington SF, Greaney ML, et al. Sociocultural and environmental influences on brazilian immigrant mothers' beliefs and practices related to child feeding and weight status. Matern Child Health J 2017;21:1085-94.

34. Lindsay AC, de Oliveira MG, Wallington SF, et al. Access and utilization of healthcare services in Massachusetts, United States: a qualitative study of the perspectives and experiences of Brazilianborn immigrant women. BMC Health Serv Res 2016;16:467.

35. Lindsay AC, Wallington SF, Greaney ML, et al. Brazilian immigrant mothers' beliefs and practices related to infant feeding: a qualitative study. J Hum Lact 2017;33.

36. Culley L, Hudson N, Rapport F. Using focus groups with minority ethnic communities: researching infertility in British South Asian communities. Qual Health Res 2007;17:102-12.

37. Kidd PS, Parshall MB. Getting the focus and the group: enhancing analytical rigor in focus group research. Qual Health Res 2000;10:293-308.

38. Faugier J, Sargeant M. Sampling hard to reach populations. J Adv Nurs 1997;26:790-7.

39. Lindsay AC, Arruda CAM, Tavares Machado MM, et al. Social contextual influences on sleep and bedtime routines among preschool-aged children of brazilian immigrant families living in the United States: a qualitative study. Children. In Press.

40. Marin G, Sabogal F, Marin BV, et al. Development of a short acculturation scale for hispanics. Hisp J Behav Sci 1987:9:183-205.

41. Miles M, Huberman A. Qualitative data analysis. 2nd edn. Thousand Oaks: Sage Publications, 1994

42. Ritchie J, Spencer L, O'Connor W. Carrying out qualitative analysis. In: Ritchie J, Lewis J, eds. Qualitative research practice. London: Sage Publications, 2004:219-62.

43. Vaismoradi $\mathrm{M}$, Turunen $\mathrm{H}$, Bondas $\mathrm{T}$. Content analysis and thematic analysis: Implications for conducting a qualitative descriptive study. Nurs Health Sci 2013;15:398-405.

44. Byrne JL, Cameron Wild T, Maximova K, et al. A brief eHealth tool delivered in primary care to help parents prevent childhood obesity: a randomized controlled trial. Pediatr Obes 2016.

45. Wright JA, Whiteley JA, Watson BL, et al. Tailored communications for obesity prevention in pediatric primary care: a feasibility study. Health Educ Res 2018;33:14-25. 
46. Janz KF, Butner KL, Pate RR. The role of pediatricians in increasing physical activity in youth. JAMA Pediatr 2013;167:595-6.

47. Sherwood NE, Levy RL, Langer SL, et al. Healthy Homes/Healthy Kids: a randomized trial of a pediatric primary care-based obesity prevention intervention for at-risk 5-10 year olds. Contemp Clin Trials 2013;36:228-43.

48. Chae J, Quick BL. An examination of the relationship between health information use and health orientation in Korean mothers: focusing on the type of health information. $J$ Health Commun $2015 ; 20: 275-84$

49. Walker LO, Mackert MS, Ahn J, et al. e-Health and new moms: contextual factors associated with sources of health information. Public Health Nurs 2017;34:561-8.

50. Kind T, Huang ZJ, Farr D, et al. Internet and computer access and use for health information in an underserved community. Ambul Pediatr 2005;5:117-21.

51. Park E, Kim H, Steinhoff A. Health-Related internet use by informal caregivers of children and adolescents: an integrative literature review. J Med Internet Res 2016;18:e57.

52. Khoo K, Bolt P, Babl FE, et al. Health information seeking by parents in the Internet age. J Paediatr Child Health 2008;44(7-8):419-23.

53. Rooks RN, Wiltshire JC, Elder K, et al. Health information seeking and use outside of the medical encounter: is it associated with race and ethnicity? Soc Sci Med 2012;74:176-84.

54. Richardson A, Allen JA, Xiao H, et al. Effects of race/ethnicity and socioeconomic status on health information-seeking, confidence, and trust. J Health Care Poor Underserved 2012;23:1477-93.

55. Clayman ML, Manganello JA, Viswanath K, et al. Providing health messages to Hispanics/Latinos: understanding the importance of language, trust in health information sources, and media use. $J$ Health Commun 2010;15:252-63.

56. Geana MV, Kimminau KS, Greiner KA. Sources of health information in a multiethnic, underserved, urban community: does ethnicity matter? J Health Commun 2011;16:583-94.

57. Standiford CJ, Nolan E, Harris M, et al. Improving the provision of language services at an academic medical center: ensuring high- quality health communication for limited-english-proficient patients. Acad Med 2009;84:1693-7.

58. Mackert M, Kahlor L, Tyler D, et al. Designing e-health interventions for low-health-literate culturally diverse parents: addressing the obesity epidemic. Telemed J E Health 2009;15:672-7.

59. Hall CM, Bierman KL. Technology-assisted interventions for parents of young children: emerging practices, current research, and future directions. Early Child Res Q 2015;33:21-32.

60. Sherwood NE, JaKa MM, Crain AL, et al. Pediatric primary carebased obesity prevention for parents of preschool children: a pilot study. Child Obes 2015;11:674-82.

61. Gorin AA, Wiley J, Ohannessian CM, et al. Steps to growing up healthy: a pediatric primary care based obesity prevention program for young children. BMC Public Health 2014;14:72.

62. Neshteruk CD, Nezami BT, Nino-Tapias G, et al. The influence of fathers on children's physical activity: a review of the literature from 2009 to 2015. Prev Med 2017;102:12-19.

63. Hillyer GC, Schmitt KM, Lizardo M, et al. Electronic communication channel use and health information source preferences among latinos in Northern Manhattan. J Community Health 2017;42:349-57.

64. Roncancio AM, Berenson AB, Rahman M. Health locus of control, acculturation, and health-related Internet use among Latinas. $J$ Health Commun 2012;17:631-40.

65. Lindsay AC, Wallington SF, Muñoz MA, et al. A qualitative study conducted in the USA exploring Latino fathers' beliefs, attitudes and practices related to their young children's eating, physical activity and sedentary behaviours. Public Health Nutr 2018;21:403-15.

66. Cloutier MM, Wiley J, Huedo-Medina T, et al. Outcomes from a pediatric primary care weight management program: steps to growing up healthy. J Pediatr 2015;167:372-7.

67. McSweeney L, Araújo-Soares V, Rapley T, et al. A feasibility study with process evaluation of a preschool intervention to improve child and family lifestyle behaviours. BMC Public Health 2017;17:248.

68. Davis AM, Daldalian MC, Mayfield CA, et al. Outcomes from an urban pediatric obesity program targeting minority youth: the Healthy Hawks program. Child Obes 2013;9:492-500. 